\title{
IS THE LATTICE OF TORSION CLASSES ALGEBRAIC?
}

\author{
JORGE MARTINEZ
}

\begin{abstract}
The answer is yes, if ...
This note attempts to give amplification to the above statement, while at the same time arriving at a reasonable description of this lattice.

The main theorem of the paper is no doubt the assertion that the lattice of torsion classes of lattice-ordered groups is completely distributive. The proof of this theorem depends on the notion of a value selector, and should not. As a consequence of this, one obtains a (local) decomposition theorem which is canonical (in every sense of the word) and always works.
\end{abstract}

The general terminology and notation of this paper is standard. It is perhaps worthwhile to make the following observations: l-group is the abbreviation for lattice-ordered group. In an l-group $G$, a value is a convex $l$-subgroup $M$, maximal with respect to the property of missing some element $x \in G$. $M$ is also said to be a value of $x$. For a reference concerning the general theory of l-groups, the reader may consult [1].

The theory of torsion classes used here is not deep. In any case, we refer the reader to [4] and [5]. In addition, we review the main definitions. A torsion class is a class of $l$-groups closed under taking (1) convex $l$-subgroups, (2) $l$ homomorphic images and (3) forming joins of convex $l$-subgroups already in the class. If $\mathcal{T}$ is a torsion class and $G$ is an $l$-group, $\mathcal{T}(G)$ stands for the join of all the convex $l$-subgroups of $G$ in $\mathcal{T} . \mathcal{T}(G)$ is a characteristic $l$-ideal, and the largest convex $l$-subgroup of $G$ in $\mathcal{T}$. It is called the torsion radical of $G$ with respect to $\mathcal{T}$.

If $\mathcal{T}$ is a torsion class we get [4, Proposition 1.1]: (a) for each convex $l$ subgroup $K$ of $G, \sigma(K)=K \cap \mathcal{T}(G)$, and (b) if $\phi: G \rightarrow H$ is an l-epimorphism, $[\mathcal{T}(G)] \Phi \subseteq \mathscr{T}(H)$. Conversely, a function subject to (a) and (b) above gives rise to a torsion class by setting $\overline{\mathfrak{T}}=\{G \mid \mathcal{T}(G)=G\}$; this correspondence between classes and radicals is one-to-one [1, Proposition 1.2].

The lattice of torsion classes-itself a proper class-is a Brouwer lattice. The meet operation is class intersection, while the join is given by: $\mathscr{T}=\vee_{i \in I} \mathscr{T}_{i}$ if and only if $\mathscr{T}(G)=\sum_{i \in I} \mathscr{T}_{i}(G)$, for each $l$-group $G$. In this note we will show this lattice to be completely distributive.

Received by the editors March 26, 1976.

AMS (MOS) subject classifications (1970). Primary 06A35, 06A60; Secondary 18E40.

Key words and phrases. Torsion class, torsion radical, value selector, algebraic lattice, compact element.

( American Mathematical Society 1977 
A homogeneous l-group $G$ is one such that $\mathcal{T}(G)=G$ or 0 , for each torsion class $\mathcal{T}$. In other words, the homogeneous $l$-groups are simple relative to their lattices of torsion radicals. Some examples of homogeneous l-groups are: cardinal sums of a fixed subgroup of the additive reals; free abelian l-groups; $C[0,1]$, the group of periodic sequences of reals; the group of bounded sequences of integers, modulo the cardinal sum.

We shall denote by $\operatorname{Rad}(G)$ the lattice of torsion radicals of $G$, by $\operatorname{Rad}$ the lattice of torsion classes, and by $M_{0}(G)$ the set of values of the l-group $G$.

1. Value selectors and complete distributivity. A value selector is a function assigning to each l-group $G$ a subset $M(G)$ of $M_{0}(G)$ subject to the conditions that: (1) if $H$ is a convex $l$-subgroup of $G$, then $M(H)=\{C \cap H \mid C$ $\in M(G), C \nsupseteq H\}$; (2) if $K$ is an $l$-ideal of $G$, then $M(G / K) \supseteq\{C / K \mid C$ $\in M(G), C \supseteq K\}$.

If $M$ is a value selector, let $\mathscr{T}_{M}=\left\{G \mid M(G)=M_{0}(G)\right\}$.

\subsection{LeMma. $\mathscr{T}_{M}$ is a torsion class.}

Proof. Suppose $H$ is a convex $l$-subgroup of $G \in \mathcal{T}_{M} \cdot M(H)=\{C$ $\left.\cap H \mid C \in M_{0}(G), H \nsubseteq C\right\}=M_{0}(H)$; i.e. $H \in \mathscr{T}_{M}$. And further, if $K$ is an l-ideal of $G \in \mathcal{T}_{M}, M(G / K) \supseteq\left\{C / K \mid C \in M_{0}(G), K \subseteq C\right\}=M_{0}(G / K)$, and so $G / K \in \mathcal{T}_{M}$.

The remaining condition is just as easy to verify, and is left to the reader.

Now let us suppose $\mathcal{T}$ is an arbitrary torsion class, and define $\hat{\mathcal{J}}(G)$ $=\left\{M \in M_{0}(G) \mid \mathcal{T}(G) \nsubseteq M\right\}$.

\subsection{LeMMA. $\hat{\Im}$ is a value selector, and $G \in \mathcal{T}$ if and only if $\hat{\mathcal{T}}(G)=M_{0}(G)$.}

Proof. The final statement of the lemma is obvious.

Suppose $H$ is a convex $l$-subgroup of $G$. If $M$ is a value of $H$, there is a unique value of $G$, say $C$, such that $C \cap H=M$. If $M \in \hat{\mathfrak{J}}(H)$ then $\mathcal{T}(H) \nsubseteq M$, and since $\widetilde{T}(H)=H \cap \mathcal{T}(G)$, this implies that $\mathscr{T}(G) \nsubseteq C$, whence $C \in \hat{\Im}(G)$. Conversely, if $M \notin \hat{\Im}(H)$, i.e. if $\mathcal{T}(H) \subseteq M$, then $\mathscr{T}(G)$ $\subseteq C$. For $C$ is the subgroup generated by those elements $0 \leqslant g \in G$ for which $g \wedge h \in M$, for all $0 \leqslant h \in H$. If $0 \leqslant g \in \mathcal{T}(G)$, then $g \wedge h$ $\in \mathcal{T}(G) \cap H=\mathscr{T}(H) \subseteq M$, for each $0 \leqslant h \in H$. It follows then that $C \notin \hat{\Im}(G)$. We can conclude therefore that $\hat{\Im}(H)=\{C \cap H \mid C \in \hat{\Im}(G), H$ $\nsubseteq C\}$.

If $K$ is an $l$-ideal of $G$, and $K \subseteq C$, with $C \in \hat{\Im}(G)$, then we wish to show that $C / K$ does not contain $\mathscr{T}(G / K)$. Select the $l$-ideal $T$ of $G$ for which $T / K=\sigma(G / K)$. If $T / K \subseteq C / K, T \subseteq C$; and since $\mathcal{T}$ is a torsion class $\mathscr{T}(G) \vee K \subseteq T$, so that $\mathscr{T}(G) \subseteq \mathcal{T}(G) \vee K \subseteq C$, a contradiction.

This proves that $\hat{\sigma}$ is a value selector, and also the lemma.

For a given value selector $M$, let $\mathbf{T}(M)=\mathscr{T}_{M}$. If $M_{1}$ and $M_{2}$ are value selectors, we define $M_{1} \leqslant M_{2}$ if $M_{1}(G) \subseteq M_{2}(G)$, for each l-group $G$. 
1.3 LEMMA. If $\mathcal{T}$ is a torsion class and $M$ is a value selector, then $\mathrm{T}(M)^{\wedge} \leqslant M$, and $\mathbf{T}(\hat{\mathrm{T}})=\sigma$.

Proof. The equation is part of Lemma 1.2; we leave the inequality as an exercise.

The containment in Lemma 1.3 can be proper: let $M$ stand for the value selector of values which are minimal prime subgroups. $\mathbf{T}(M)$ is then the torsion class of hyperarchimedean $l$-groups. However $\mathbf{T}(M)^{\wedge}$ need not select all values that are minimal primes. It is known, for example, that the free abelian l-group on two generators has the property that every prime subgroup is either maximal or minimal [2]; yet the hyperarchimedean radical of such a group is 0 .

1.4 Lemma. Suppose $\left\{\mathscr{T}_{\lambda} \mid \lambda \in \Lambda\right\}$ is a family of torsion classes. Then $\left(\cap_{\lambda \in \Lambda} \widetilde{T}_{\lambda}\right)^{\hat{\prime}}=\Lambda_{\lambda \in \Lambda} \hat{\widetilde{T}}_{\lambda}$, and $\left(V_{\lambda \in \Lambda} \tilde{T}_{\lambda}\right)^{\hat{\prime}}=V_{\lambda \in \Lambda} \hat{\tilde{T}_{\lambda}}$.

Note. The lattice operations on value selectors are to be interpreted in the only way possible: thus, if $\left\{M_{i} \mid i \in I\right\}$ is a family of value selectors, $\left(\wedge_{i \in I} M_{i}\right)(G)=\bigcap_{i \in I} M_{i}(G)$; and $\left(\vee_{i \in I} M_{i}\right)(G)=\cup_{i \in I} M_{i}(G)$.

Proof of Lemma 1.4. Let $G$ be an arbitrary l-group. $M \in\left(\vee_{\lambda \in \Lambda} \mathscr{T}_{\lambda}\right) \hat{}(G)$ if and only if $M \nsupseteq \sum_{\lambda \in \Lambda} \sigma_{\lambda}(G)$; i.e. if and only if $M \nsupseteq \sigma_{\mu}(G)$, for some $\mu \in \Lambda$. Thus, $M \in\left(\bigvee_{\lambda \in \Lambda} \widetilde{T}_{\lambda}\right)^{\wedge}(G)$ if and only if $M \in \cup_{\lambda \in \Lambda} \sigma_{\lambda}{ }^{\wedge}(G)$.

On the other hand, $M \in\left(\cap_{\lambda \in \Lambda} \mathcal{T}_{\lambda}\right)^{\wedge}(G)$ exactly when $\bigcap_{\lambda \in \Lambda} \mathcal{T}_{\lambda}(G) \nsubseteq M$. This occurs precisely when $\sigma_{\lambda}(G) \nsubseteq M$ for all $\lambda \in \Lambda$. This is where we use the meet irreducibility of values in the lattice of convex $l$-subgroups of $G$; curiously, it is the only place. Hence, $M \in\left(\cap_{\lambda \in \Lambda} \mathcal{T}_{\lambda}\right)^{\wedge}(G)$ if and only if $M \in \wedge_{\lambda \in \Lambda} \hat{\tilde{T}}_{\lambda}(G)$.

This completes the proof of the lemma.

1.5 THEOREM. The assignment $\mathcal{T} \rightarrow \hat{\mathfrak{T}}$ embeds the lattice $\mathrm{Rad}$ of torsion classes as a complete sublattice of the lattice of value selectors. Since the latter is completely distributive, so is $\mathrm{Rad}$.

Comments. A. The lattice of value selectors is completely distributive, since it is, locally, a lattice of subsets.

B. For any torsion class $\mathcal{T}$, it should be clear that $\mathscr{T}(G)=0$ if and only if $\hat{\jmath}(G)=\varnothing$.

C. The function $M \rightarrow \mathbf{T}(M)$ also preserves arbitrary intersections. This is rather easy to prove, and we shall omit it here. But it is unknown whether it also preserves joins. It would be of interest to know this, for it would shed light on the following question: If $\mathcal{T}$ is a torsion class, is there a largest value selector $M$ such that $\mathbf{T}(M)=\sigma$ ? There is always a smallest, namely $\hat{\jmath}$. In view of the inequality in Lemma 1.3, the author doubts that it preserves joins.

D. It is immediate from the definition of $\hat{\mathfrak{T}}$, once a torsion class $\mathfrak{T}$ is given, that $\hat{\mathscr{T}}(G)$ is an ideal of values of the l-group $G$. That is: if $M \in \hat{\mathfrak{T}}(G)$ and $N \subseteq M, N \in M_{0}(G)$, then $N \subset \hat{\Im}(G)$. This however does not characterize the $\hat{\mathcal{T}}$. For the value selector of minimal primes gives an ideal of each $M_{0}(G)$, 
yet it is not the smallest value selector associated with hyperarchimedean $l$ groups.

The upshot of Theorem 1.5 is that $\operatorname{Rad}(G)$ is a completely distributive, algebraic lattice. It is valuable therefore to find out some information about the compact elements of this lattice, i.e. the finitely generated torsion radicals. (Abstractly, the definition of compactness of $c$ in a lattice $L$ is as follows: $c \leqslant \vee_{\lambda \in \Lambda} x_{\lambda}$ implies that $c \leqslant x_{\lambda_{1}} \vee \cdots \vee x_{\lambda_{k}}$, for certain $\lambda_{1}, \lambda_{2}, \ldots, \lambda_{k}$ $\in \Lambda$.) For we can now apply the main theorem in [3] directly to prove a canonical decomposition theorem for $l$-groups. The theorem of [3] in question can be stated as follows.

1.6 THEOREM. If $L$ is an algebraic lattice, the following are equivalent statements. $C$ denotes the join-subsemilattice of compact elements.

(i) $L$ is completely distributive;

(ii) each meet irreducible element $m \in L$ is maximal with respect to not exceeding a finitely join irreducible element in $C$;

(iii) each $x \in C$ has a unique decomposition $x=x_{1} \vee x_{2} \vee \cdots \vee x_{n}$ in $C$, where each $x_{i}$ is finitely join-irreducible, and any two are pairwise incomparable.

Applying 1.6 directly to $\operatorname{Rad}(G)$, when $G$ has a strong order unit (an element $0<u \in G$ with the property that for each $0 \leqslant g \in G$ there is a positive integer $n$ such that $g \leqslant n u$ ), we obtain

1.7 THEOREM. If $G$ has a strong order unit, we can write $G$ uniquely as follows: $G=\mathscr{T}_{1}(G)+\mathcal{T}_{2}(G)+\cdots+\mathcal{T}_{n}(G)$, where the $\mathcal{T}_{i}(G)$ are finitely generated, finitely join irreducible torsion radicals, and no two are comparable.

REMARK. Although we shall not prove it here, it can be shown that with regard to the statement of Theorem 1.6, the decomposition in (iii) is directmeaning that $x_{i} \wedge x_{j}=0$, if $i \neq j$-if and only if the set of meet irreducible elements forms a root system. (Recall: a root system is a p.o. set $P$ in which $\{x \in P \mid x \geqslant a\}$ is a chain for each $a \in P$.)

In the situation with $\operatorname{Rad}(G)$ one can easily manufacture an example for $G$ so that the decomposition in Theorem 1.7 is not direct. In general then, the meet irreducible torsion radicals do not form a root system.

2. And now: algebraic or no! Let us turn to Rad itself. The following statements all have easy verifications:

2.1. If a torsion class $\mathcal{T}$ can be generated by a set of $l$-groups it can be generated by their cardinal sum. Thus, torsion classes can be singly generated precisely when they can be generated by a set of $l$-groups.

2.2. Suppose $\mathfrak{X}=\mathfrak{X}_{G}$, the torsion class generated by the l-group $G$. The $l$ groups of $\mathscr{X}$ (see [5]) are those which are sums of convex $l$-subgroups which are themselves isomorphic to subquotients of $G$. (A subquotient of $G$ is an $l$ homomorphic image of a convex $l$-subgroup of $G$.) From this observation follow: 
(a) Each torsion subclass of $\mathcal{X}$ is generated by a set, and hence by one $l$ group.

(b) If $\left\{G_{i} \mid i \in I\right\}$ is a class of $l$-groups generating a torsion class $\mathcal{T}$, then $\mathscr{T}=\bigvee_{i \in I} \mathfrak{X}_{G_{i}}$.

2.3. If $\mathcal{X}=\mathcal{X}_{G}$ and $G$ has a strong order unit, then it is easily shown that $\mathcal{X}$ is compact in $\mathrm{Rad}$.

Conversely, suppose $\mathcal{X}$ is a compact torsion class; by 2.2(b) $X=\bigvee_{i \in I} X_{G_{i}}$, for generating l-groups $G_{i}$. Without loss of generality, each $G_{i}$ can be assumed to possess a strong order unit. (Simply write each $G_{i}$ as the union of its principal convex $l$-subgroups. $G_{i}$ generates the join of the classes each one of these generate.) By the compactness of $\mathcal{X}, \mathcal{X}=\mathscr{X}_{G_{1}} \vee \cdots \vee \mathscr{X}_{G_{k}}$, for suitable $G_{1}, G_{2}, \ldots, G_{k}$, appropriately relabeled. If $G$ denotes the cardinal sum of $G_{1}, G_{2}, \ldots, G_{k}$ then $\mathcal{X}=\mathscr{X}_{G}$, and $G$ has a strong order unit. Thus:

2.4 Proposition. In Rad, the compact elements are those torsion classes which can be generated by one l-group with a strong order unit.

Suppose $G$ is an l-group with a strong unit, and $\mathcal{X}=\mathfrak{X}_{G}$. According to Theorem 1.7, $G$ can be written: $G=T_{1}+\cdots+T_{n}$, where the $T_{i}$ are the canonical finitely join irreducible components in $\operatorname{Rad}(G)$. Suppose $U_{1}, U_{2}$, $\ldots, U_{n}$ are the maximal torsion radicals of $G$, arranged so that $U_{i}$ is maximal with respect to not containing $T_{i}$.

2.5. Each $G / U_{i}(1 \leqslant i \leqslant n)$ is a homogeneous l-group.

Now for each $U_{i}$ let $\mathcal{U}_{i}(1 \leqslant i \leqslant n)$ be the largest torsion subclass of $\mathfrak{X}$ such that $\mathscr{U}_{i}(G)=U_{i}$, and for each $T_{i}$ let $\mathcal{T}_{i}(1 \leqslant i \leqslant n)$ be the smallest torsion class such that $\mathcal{T}_{i}(G)=T_{i}$.

The following proposition is then easy to verify.

2.6 Proposition. If $G$ has a strong order unit and $\mathcal{X}=\mathfrak{X}_{G}$, then $\mathcal{X}$ $=\sigma_{1} \vee \sigma_{2} \vee \cdots \vee \mathcal{T}_{n}$, with $\mathcal{T}_{i}$ 's as defined above. Each $\mathcal{T}_{i}$ is compact in Rad, and also finitely join irreducible.

Finally, the $\mathscr{Q}_{i}(1 \leqslant i \leqslant n)$ are precisely the maximal torsion subclasses of X.

In short then, $\operatorname{Rad}$ imitates the structure of each $\operatorname{Rad}(G)$ rather nicely. So again the question: Is Rad algebraic?

Without pretension, we would like to culminate this article with an amusing subtlety. Consider: in a lattice $L, c \in L$, is compact if $c \leqslant V_{\lambda \in \Lambda} x_{\lambda}$ implies $c \leqslant x_{\lambda_{1}} \vee x_{\lambda_{2}} \vee \cdots \vee x_{\lambda_{k}}$, for suitable $\lambda_{1}, \lambda_{2}, \ldots, \lambda_{k} \in \Lambda$. And $L$ is algebraic provided each element is the join of compact elements.

If in the above definitions one permits families indexed over a class, or considers elements as a join of possibly a class of compact elements, then according to Proposition 2.4, the compact elements of $\mathrm{Rad}$ are precisely the torsion classes generated by a single l-group with a strong order unit. Further, by Proposition 2.2(b) each torsion class is the join of compact classes, whence $\mathrm{Rad}$ is algebraic. 
However, suppose one insists on only allowing sets of indices and joins of sets of compact classes. The torsion classes generated by one l-group with a strong order unit are still compact in Rad. A join irreducible class is compact, and join irreducibility here means: $x=\bigvee_{\lambda \in \Lambda} x_{\lambda}$ implies that $x=x_{\mu}$ for some $\mu \in \Lambda$, whenever $\Lambda$ is a set.

A torsion class $\mathscr{T}$ is complete if it is closed under extensions: i.e. if $G$ is an $l$ group and $A$ is an $l$-ideal of $G$ so that $A$ and $G / A$ are in $\mathscr{J}$, then so is $G$. In [4, Theorem 1.6], it was shown that each torsion class can be embedded in a unique minimal complete torsion class. It can be shown, using wreath products, that any complete class is join irreducible. This result will appear in due course, and is due to Charles Holland and the author. It follows then that every torsion class is contained in one which is compact.

Obviously, this falls short of deciding whether Rad is algebraic in this restricted sense.

\section{REFERENCES}

1. P. Conrad, Lattice-ordered groups, Tulane Univ., 1970.

2. _— Epi-archimedean groups, Czechoslovak Math. J. 24 (99) (1974), 192-218. MR 50 \#203.

3. J. Martinez, Unique factorization in partially ordered sets, Proc. Amer. Math. Soc. 33 (1972), 213-220. MR 45 \#1806.

4. — Torsion theory for lattice-ordered groups, Czechoslovak Math. J. 25 (100) (1975), 284-299.

5. - Torsion theory for lattice-ordered groups. II, Czechoslovak Math. J. 26 (101) (1976), 93-100.

Department of Mathematics, University of Florida, Gainesville, Florida 32611 